\title{
Boundary procedures for the time-dependent Burgers' equation under uncertainty
}

Per Pettersson, Jan Nordström and Gianluca Iaccarino

\section{Linköping University Post Print}

N.B.: When citing this work, cite the original article.

Original Publication:

Per Pettersson, Jan Nordström and Gianluca Iaccarino, Boundary procedures for the timedependent Burgers' equation under uncertainty, 2010, Acta Mathematica Scientia, (30), 539550 .

http://dx.doi.org/10.1016/S0252-9602(10)60061-6

Copyright: Elsevier Science B.V. Amsterdam

http://www.elsevier.com/

Postprint available at: Linköping University Electronic Press

http://urn.kb.se/resolve?urn=urn:nbn:se:liu:diva-68607 


\title{
Boundary Procedures for the Time-dependent Burgers' Equation Under Uncertainty
}

\author{
Per Pettersson, Jan Nordström and Gianluca Iaccarino \\ Stanford University
}

\begin{abstract}
The Burgers' equation with uncertain initial and boundary conditions is approximated using a Polynomial Chaos Expansion (PCE) approach where the solution is represented as a series of stochastic, orthogonal polynomials. The resulting truncated PCE system is solved using a novel numerical discretization method based on spatial derivative operators satisfying the summation by parts property and weak boundary conditions to ensure stability. The resulting PCE solution yields an accurate quantitative description of the stochastic evolution of the system, provided that appropriate boundary conditions are available. The specification of the boundary data is shown to influence the solution; we will discuss the problematic implications of the lack of precisely characterized boundary data and possible ways of imposing stable and accurate boundary conditions.
\end{abstract}

Key words Stochastic Burgers' Equation, Uncertainty Quantification, Polynomial Chaos

\section{Introduction}

In many physical problems our knowledge is limited by our ability to measure, by our bias in the observations and, in general, by an incomplete understanding of the relevant processes. When we attempt to construct a mathematical representation of the problem, we must account for those limitations and, in addition, we must identify the possible limitations of the numerical techniques and phenomenological models that we employ.

Numerical simulations are subject to uncertainty in boundary or initial conditions, model parameter values and even in the geometry of the physical domain of interest; this results in uncertainty in the output data that must be clearly identified and quantified. Fields of application of uncertainty quantification include but are not limited to turbulence, climatology [13], combustion [14], flow in porous media [4], fluid mixing [15] and computational electromagnetics [3].

The problem considered in this paper is the characterization of the uncertainty in the dynamics of a shock wave induced by lack of precise information regarding the

Submitted November 2009. Supported by the US Department of Energy under the PSAAP Program. 
initial and boundary conditions. We perform an uncertainty quantification analysis for the stochastic Burgers' equation by employing a spectral representation of the solution in the form of polynomial chaos expansion [5]. The equation is stochastic as a result of uncertainty in the initial and boundary values which are represented using random variables. The stochastic Galerkin projection of the stochastic Burgers' equation results in a deterministic system of equations from which the expected values, the variance and high-order moments of the solution can be readily determined.

The application of the polynomial chaos approach leads to a new hyperbolic systems with multiple discontinuities [12]. The weak resemblance to the corresponding deterministic problem suggests an appropriate way to specify boundary conditions for the solution mean, but gives no concrete information on the treatment of higher order moments.

Due to limited information on boundary data as well as to the computational cost of high order polynomial chaos simulations, low-order approximations are typically employed in practice. In this paper we will investigate the accuracy of low order approximations, particularly when appropriate high order boundary data are missing. Because of the hyperbolic nature of the problem, information is traveling with finite but unknown speed through the domain and will eventually affect the boundary. By the convergence properties of the polynomial chaos series expansion, higher order boundary terms are expected to decrase rapidly. On the other hand, although small, these coefficient have a relatively large impact on the systen eigenvalues and might thus be crucial for accurate boundary treatment. In addition to this, there are discontinuities in the stochastic dimension (we only assume one stochastic dimension), which deteriorates the convergence. The net effect of the higher order boundary coefficients is not clear and motivates the investigation of this paper.

\section{Polynomial chaos expansion of Burgers' equation}

We consider the Burgers' equation,

$$
u_{t}+u u_{x}=0,0 \leq x \leq 1
$$

and assume that the uncertainty in the initial or the boundary conditions is represented in terms of a random variable $\xi \in \Omega$. The solution $u(x, t, \xi)$ is described by an infinite series in an orthogonal polynomial basis (polynomial chaos representation):

$u(x, t, \xi)=\sum_{i=0}^{\infty} u_{i} \Psi_{i}(\xi)$ that inserted into the Burgers' equation yields a system of coupled equations:

$$
\sum_{i=0}^{\infty} \frac{\partial u_{i}}{\partial t} \Psi_{i}(\xi)+\left(\sum_{j=0}^{\infty} u_{j} \Psi_{j}(\xi)\right)\left(\sum_{i=0}^{\infty} \frac{\partial u_{i}}{\partial x} \Psi_{i}(\xi)\right)=0
$$


A stochastic Galerkin projection is performed by multiplying (2) by $\Psi_{k}(\xi)$ for nonnegative integers $k$ and integrating over the probability domain $\Omega$. Using the orthogonality of the basis polynomials we obtain a system of deterministic equations. By truncating the number of polynomial chaos coefficients to a finite number $M$, the solution is projected onto a finite dimensional space. The result is a symmetric system of equations.

$$
\frac{\partial u_{k}}{\partial t}\left\langle\Psi_{k}^{2}\right\rangle+\sum_{i=0}^{M} \sum_{j=0}^{M} u_{i} \frac{\partial u_{j}}{\partial x}\left\langle\Psi_{i} \Psi_{j} \Psi_{k}\right\rangle=0 \quad \text { for } k=0,1, \ldots, M .
$$

For simplicity of notation, equation (3) can be written in matrix form as

$$
B u_{t}+A(u) u_{x}=0 \quad \text { or } \quad B u+\frac{1}{2} \frac{\partial}{\partial x}(A(u) u)=0
$$

which are the forms that will be used in the following sections.

As an illustration, the $3 \times 3$ system obtained by truncating the expansion to $M=2$ with a Hermite polynomial basis for Burgers' equation is

$$
\left(\begin{array}{lll}
1 & 0 & 0 \\
0 & 1 & 0 \\
0 & 0 & 2
\end{array}\right)\left(\begin{array}{l}
u_{0} \\
u_{1} \\
u_{2}
\end{array}\right)_{t}+\left(\begin{array}{ccc}
u_{0} & u_{1} & 2 u_{2} \\
u_{1} & u_{0}+2 u_{2} & 2 u_{1} \\
2 u_{2} & 2 u_{1} & 2 u_{0}+8 u_{2}
\end{array}\right)\left(\begin{array}{l}
u_{0} \\
u_{1} \\
u_{2}
\end{array}\right)_{x}=0
$$

Note that the matrix $A(u)$ is symmetric and that the "mass matrix" $B$ is diagonal.

\section{Problem setup}

In order to quantify the accuracy of the results computed using the polynomial representation of the solution as a function of the order of truncation $M$, we formulate a test with an analytical solution. Consider the stochastic Riemann problem with an initial shock location $x_{0} \in \Omega$

$$
\begin{aligned}
& u(x, 0, \xi)=\left\{\begin{array}{l}
u_{L}=a+P(\xi) \text { if } x<x_{0} \\
u_{R}=-a+P(\xi) \text { if } x>x_{0}
\end{array}\right. \\
& u(0, t, \xi)=u_{L}, u(1, t, \xi)=u_{R} \\
& \xi \in N(0,1),
\end{aligned}
$$

As the most intuitive choice of polynomial basis with regard to the boundary uncertainty, the set of Hermite polynomials will be used [5]. Here we will only consider 
$P(\xi)=b \xi$ and both $a$ and $b$ be known constant. By the Rankine-Hugoniot condition, the shock speed is given by $s=P(\xi)$ and the shock location $x_{s}$ is

$$
x_{s}=x_{0}+t P(\xi)
$$

The solution is given by

$$
u(x, t, \xi)=\left\{\begin{array}{l}
u_{L} \text { if } x<x_{0}+t P(\xi) \\
u_{R} \text { if } x>x_{0}+t P(\xi)
\end{array}\right.
$$

and is uniquely defined by the countable set of polynomial chaos coefficients $\left\{u_{0}, u_{1}, \ldots\right\}$ where

$$
u_{i}(x, t)=\frac{1}{\left\langle\Psi_{i}^{2}\right\rangle} \int_{-\infty}^{\infty} u(x, t, \xi) \Psi_{i}(\xi) f(\xi) d \xi
$$

An analytical solution to the stochastic problem above can be derived by using the deterministic techniques for Riemann problems. In a previous paper [12], we studied the dependency of the solution on the order of truncation; here we will consider expansions of order $M=1$. Expectation and variance can be expressed in terms of the polynomial chaos coefficients, as

$$
\mathbf{E}(u)=u_{0}
$$

and

$$
\operatorname{Var}(u)=\sum_{i=1}^{\infty} u_{i}\left\langle\Psi_{i}^{2}\right\rangle
$$

respectively. Clearly, $\mathbf{E}(u)$ (i.e. $u_{0}$ ) will be available (however distorted) no matter of the order of truncation of the system whereas only the first few coefficients will be used to approximate the variance.

\section{Well posedness and stability}

In order to ensure stability of the discretized system of equations, summation by parts operators and weak imposition of boundary conditions $[1,10,11,2]$ are used to obtain energy estimates. The system is expressed in a split form that combines the conservative and non-conservative formulation [9]. A particular set of artificial dissipation operators [8] are used to enhance the stability close to the shock. Burgers' equation has been discretized with a fourth order central difference operator in space and the fourth order Runge-Kutta method in time. For stability, artificial dissipation is added based on the local system eigenvalues. The order of accuracy is not affected by the addition of artificial dissipation. 
The dominating error is instead due to truncation of the polynomial chaos expansion. General difficulties related to solving hyperbolic problems and nonlinear conservation laws with spectral methods are discussed in $[6,12]$. Here we investigate well posedness and stability.

A problem is well posed [7, 9] if the solution exists, is unique and depends continuously on the problem data. The system is written in split form as

$$
H u_{t}+\beta \frac{\partial}{\partial x}\left(\frac{A}{2} u\right)+(1-\beta) A u_{x}=0,0 \leq x \leq 1
$$

The solution is assumed to be smooth. After multiplication by $u^{T}$ and integration by parts, we obtain

$$
\frac{1}{2} \frac{\partial}{\partial t}\|u\|_{H}^{2}=-\frac{\beta}{2}\left[u^{T} A u\right]_{x=0}^{x=1}+\frac{\beta}{2} \int_{0}^{1} u_{x}^{T} A u d x-(1-\beta) \int_{0}^{1} u^{T} A u_{x} d x,
$$

where we choose $\beta=2 / 3$. The energy method yields an energy estimate of the form

$$
\|u\|_{\Omega}^{2}+\frac{2}{3} \int_{0}^{t}\left\|w_{0}\right\|_{\Gamma}^{2}+\left\|w_{1}\right\|_{\Gamma}^{2} d \tau \leq\|f\|_{\Omega}^{2}+\frac{4}{3} \int_{0}^{t}\left\|g_{0}\right\|_{\Gamma}^{2}+\left\|g_{1}\right\|_{\Gamma}^{2} d \tau
$$

Since $w=V^{-1} u$ and $\|w\| \leq\left\|V^{-1}\right\|\|u\| \leq C\|u\|$ for some $C<\infty$, the estimate (10) leads to strong well-posedness.

To obtain stability, we will use the so-called penalty technique [8] to impose boundary conditions for the discrete problem [12]. Let $E_{0}=\left(e_{i j}\right)$ where $e_{11}=1, e_{i j}=$ $0, \forall i, j \neq 1$ and $E_{n}=\left(e_{i j}\right)$ where $e_{n n}=1, e_{i j}=0, i, j \neq n$. Define the block diagonal matrix $A_{g}$ where the diagonal blocks are the symmetric matrices $A(u(x))$. With penalty matrices $\Sigma_{0}$ and $\Sigma_{1}$ corresponding to the left and right boundaries respectively, the discretized system can be expressed as

$$
(I \otimes H) u_{t}+A_{g}\left(P^{-1} Q \otimes I\right) u=\left(P^{-1} \otimes I\right)\left(E_{0} \otimes \Sigma_{0}\right)\left(u-g_{0}\right)+\left(P^{-1} \otimes I\right)\left(E_{n} \otimes \Sigma_{1}\right)\left(u-g_{1}\right) .
$$

Similarly, the conservative system in (4) can be discretized as

$$
(I \otimes B) u_{t}+\frac{1}{2}\left(P^{-1} Q \otimes I\right) A_{g} u=\left(P^{-1} \otimes I\right)\left(E_{0} \otimes \Sigma_{0}\right)\left(u-g_{0}\right)+\left(P^{-1} \otimes I\right)\left(E_{n} \otimes \Sigma_{1}\right)\left(u-g_{1}\right) .
$$

A linear combination of the conservative and the non-conservative form is used for the energy estimates, just as in the continuous case. The split form is given by

$$
\begin{aligned}
(I \otimes H) u_{t}+\beta \frac{1}{2}\left(P^{-1} Q \otimes I\right) A_{g} u+(1-\beta) A_{g}\left(P^{-1} Q \otimes I\right) u & = \\
& =\left(P^{-1} \otimes I\right)\left[\left(E_{0} \otimes \Sigma_{0}\right)\left(u-g_{0}\right)+\left(E_{n} \otimes \Sigma_{1}\right)\left(u-g_{1}\right)\right] .
\end{aligned}
$$


Multiplication by $u^{T}(P \otimes I)$ and then addition of the transpose of the resulting equation yields

$$
\begin{aligned}
\frac{\partial}{\partial t}\|u\|_{(P \otimes H)}^{2}+\frac{\beta}{2} u^{T}\left((Q \otimes I) A_{g}+A_{g}\left(Q^{T} \otimes I\right)\right) u+ & \\
+(1-\beta) & u^{T}\left(A_{g}(Q \otimes I)+\left(Q^{T} \otimes I\right) A_{g}\right) u= \\
& =2 u^{T}\left(E_{0} \otimes \Sigma_{0}\right)\left(u-g_{0}\right)+2 u^{T}\left(E_{n} \otimes \Sigma_{1}\right)\left(u-g_{1}\right) .
\end{aligned}
$$

With the choice $\beta=2 / 3$, the energy methods yields

$\frac{\partial}{\partial t}\|u\|_{(P \otimes B)}^{2}=\frac{2}{3}\left(u_{x=0}^{T} A u_{x=0}-u_{x=1}^{T} A u_{x=1}\right)+2 u_{x=0}^{T} \Sigma_{0}\left(u_{x=0}-g_{0}\right)+2 u_{x=1}^{T} \Sigma_{1}\left(u_{x=1}-g_{1}\right)$.

Restructuring (15) yields

$\frac{\partial}{\partial t}\|u\|_{(P \otimes B)}^{2}=u_{x=0}^{T}\left(\frac{2}{3} A+2 \Sigma_{0}\right) u_{x=0}-2 u_{x=0}^{T} \Sigma_{0} g_{0}-u_{x=1}^{T}\left(\frac{2}{3} A-2 \Sigma_{1}\right) u_{x=1}-2 u_{x=1}^{T} \Sigma_{1} g_{1}$.

Stability is achieved by a proper choice of the penalty matrices $\Sigma_{0}$ and $\Sigma_{1}$. For that purpose $A$ is split according to the sign of its eigenvalues as

$$
A=A^{+}+A^{-} \text {where } A^{+}=x^{T} \Lambda^{+} x \text { and } A^{-}=x^{T} \Lambda^{-} x .
$$

Choose $\Sigma_{0}$ and $\Sigma_{1}$ such that $\frac{2}{3} A^{+}+2 \Sigma_{0}=-\frac{2}{3} A^{+} \Leftrightarrow \Sigma_{0}=-\frac{2}{3} A^{+}$and $\frac{2}{3} A^{-}-2 \Sigma_{1}=$ $\frac{2}{3} A^{-} \Leftrightarrow \Sigma_{1}=\frac{2}{3} A^{-}$. We now get the energy estimate

$$
\begin{aligned}
\frac{\partial}{\partial t}\|u\|_{(P \otimes B)}^{2} & =-\frac{2}{3}\left(u_{x=0}-g_{0}\right)^{T} A^{+}\left(u_{x=0}-g_{0}\right)+\frac{2}{3}\left[u_{x=0}^{T} A^{-} u_{x=0}+g_{0}^{T} A^{+} g_{0}\right] \\
- & \frac{2}{3}\left[u_{(x=1)}^{T} A^{+} u_{(x=1)}+g_{1}^{T} A^{-} g_{1}\right]+\frac{2}{3}\left(u_{(x=1)}-g_{1}\right)^{T} A^{-}\left(u_{(x=1)}-g_{1}\right),
\end{aligned}
$$

which shows that the system is stable.

In the short summary of well-posedness and stability analysis above we have assumed that we have perfect knowledge of boundary data but this is rarely true. In practical calculations lack of data makes such analysis impossible and one has to rely on estimates to assign boundary data. We will investigate the effect of that problem next.

\section{Dependence on available data}

For $M=1$, the system (4) can be diagonalized with constant eigenvectors and we get an exact solution to the truncated problem. With $a$ and $b$ as in the problem setup, the 
analytical solution for the $2 \times 2$-system $(x \in[0,1])$ is given by

$$
\left(\begin{array}{l}
u_{0} \\
u_{1}
\end{array}\right)= \begin{cases}(a, b)^{T} & \text { if } x<x_{0}-b t \\
(0, a+b)^{T} & \text { if } x_{0}-b t<x<x_{0}+b t \\
(-a, b)^{T} & \text { if } x>x_{0}+b t \\
(0, a+b)^{T} & \text { for } 0 \leq t<\frac{x_{0}}{b}\end{cases}
$$

We expect different numerical solutions depending on the amount of available boundary data. We will assume that the boundary data are known on the boundary $x=1$ and investigate three different cases for the left boundary $x=0$ corresponding to complete set of data, partial information about boundary data and no data available, respectively. For all cases, we will solve a system of the form

$$
\left(\begin{array}{l}
u_{0} \\
u_{1}
\end{array}\right)_{t}+\frac{1}{2}\left[\left(\begin{array}{ll}
u_{0} & u_{1} \\
u_{1} & u_{0}
\end{array}\right)\left(\begin{array}{l}
u_{0} \\
u_{1}
\end{array}\right)\right]_{x}=0 .
$$

with boundary data

$$
\left(\begin{array}{l}
u_{0} \\
u_{1}
\end{array}\right)_{x=-1}=\left(\begin{array}{l}
g_{0}(t) \\
g_{1}(t)
\end{array}\right) ;\left(\begin{array}{l}
u_{0} \\
u_{1}
\end{array}\right)_{x=1}=\left(\begin{array}{l}
h_{0}(t) \\
h_{1}(t)
\end{array}\right) .
$$

\subsection{Complete set of data}

The boundary conditions are

$$
u(0, t)=\left\{\begin{array}{l}
(a, b)^{T} \quad 0 \leq t<\frac{x_{0}}{b} \\
(0, a+b)^{T} t>\frac{x}{b}
\end{array}\right.
$$

Consider $a=1, b=0.2$. Both $u_{0}$ and $u_{1}$ are known at $x=0$ and the two ingoing char-

acteristics are assigned the analytical values. The system satisfies the energy estimate (18) and is stable. Figure 1,2 and 3 show the solution at time $t=1, t=2$ and $t=3$ respectively. 

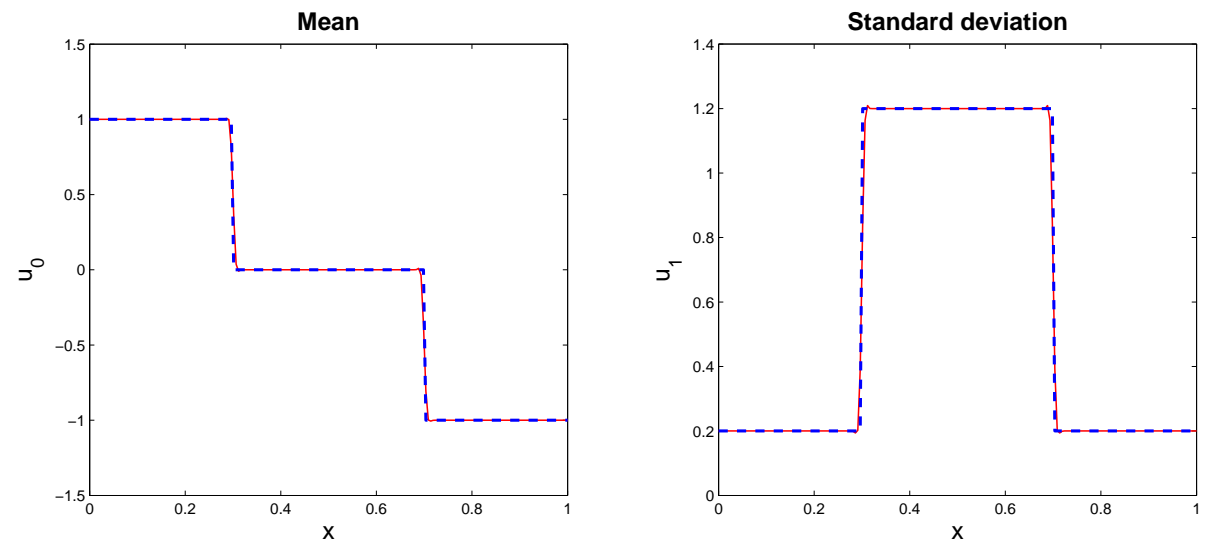

Figure 1: $u_{0}$ (left) and $u_{1}$ (right). $t=1$. Complete set of data. Analytical solution: ---- ; Numerical solution: — .
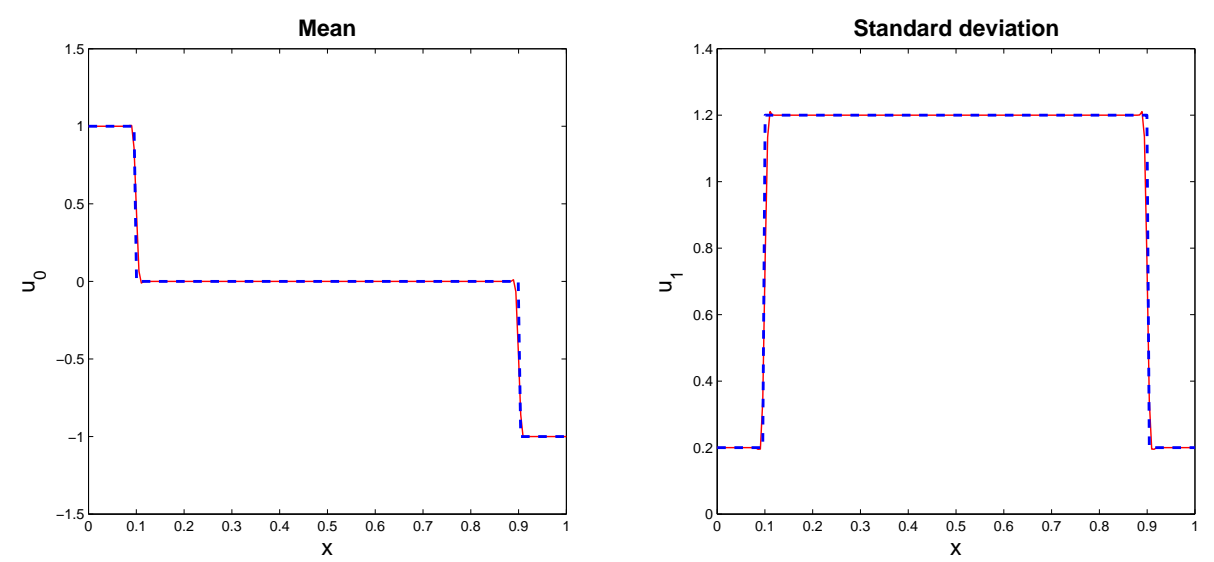

Figure 2: $u_{0}$ (left) and $u_{1}$ (right). $t=2$. Complete set of data. Analytical solution: ---- ; Numerical solution: - 

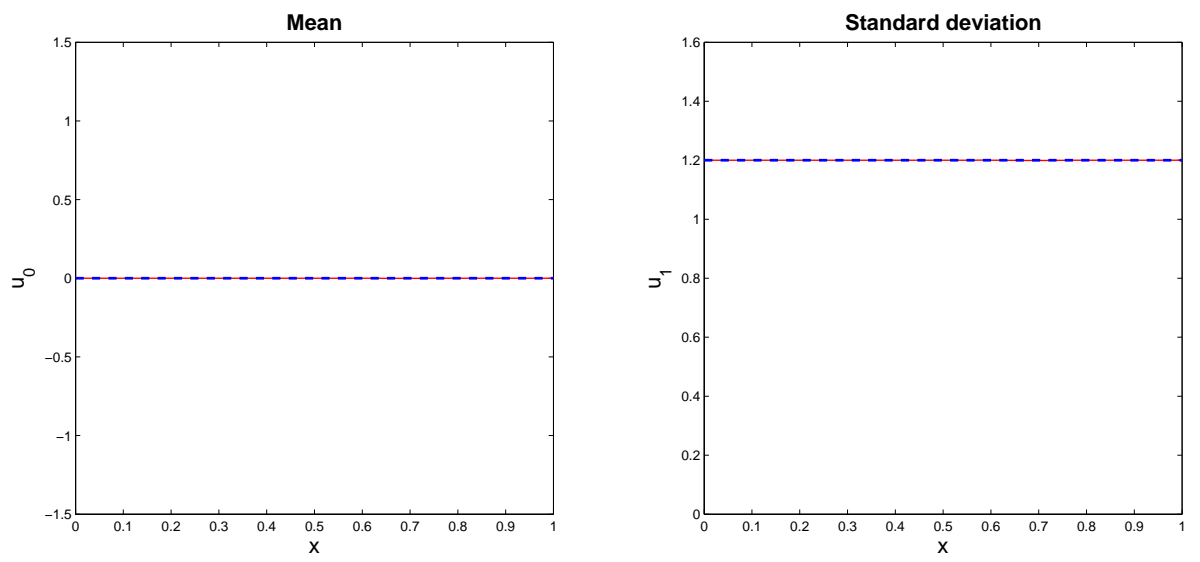

Figure 3: $u_{0}$ (left) and $u_{1}$ (right). $t=3$. Complete set of data. Analytical solution: ---- ; Numerical solution: -

\subsection{Incomplete set of boundary data}

Without a complete set of boundary data, the time-dependent behavior of the solution will be hard to predict. Here we assume that the boundary conditions at $x=1$ is $u=(-1,0.2)$ as before (Equation $(21))$ and consider different ways of dealing with unknown data at $x=0$. The initial function is the same as in the analytical problem above, i.e.

$$
\left(u_{0}(x, 0), u_{1}(x, 0)\right)^{T}= \begin{cases}(a, b)^{T} & \text { if } x<x_{0} \\ (-a, b)^{T} & \text { if } x>x_{0}\end{cases}
$$

\subsection{1 $u_{1}$ unknown at $x=0$, guess $u_{1}$}

First assume that $u_{0}$ is known and $u_{1}$ is unknown and set $u_{1}=0.2$ at the boundary for all time. This problem setup lead to an energy estimate and stability. There are two ingoing characteristics at $t=0 . u_{0}$ at $x=0$ changes with the boundary conditions as given by (21). The time development follows the analytical solution at first (Figure 4) but eventually becomes inconsistent with the boundary conditions (Figure 5 and Figure 6) 

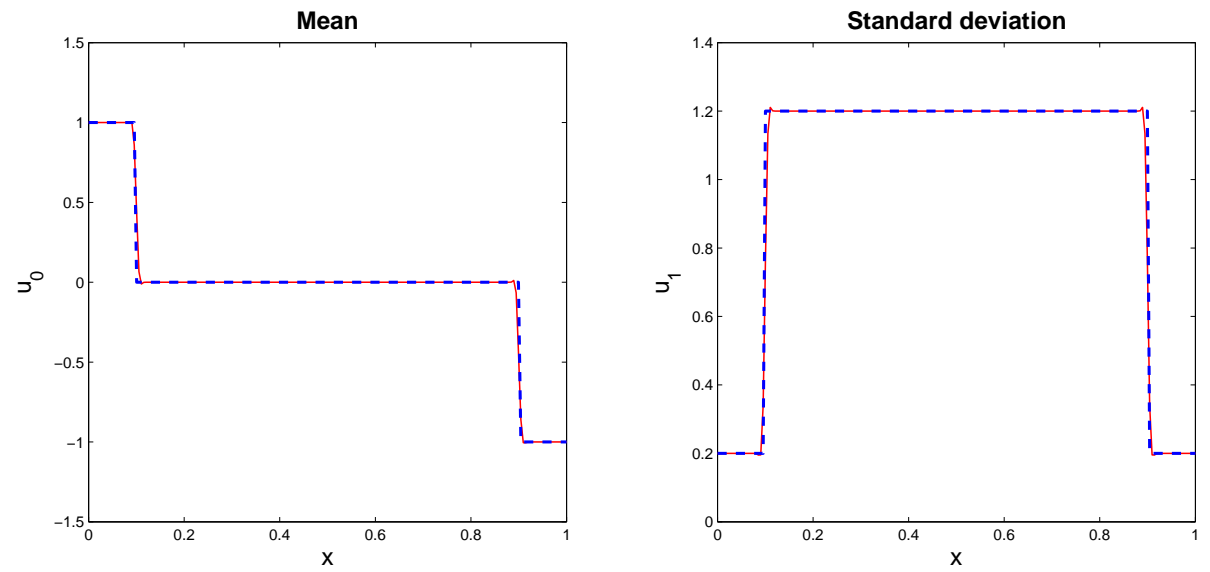

Figure 4: $u_{1}$ kept fixed at 0.2. $t=2$. Analytical solution: ---- ; Numerical solution:
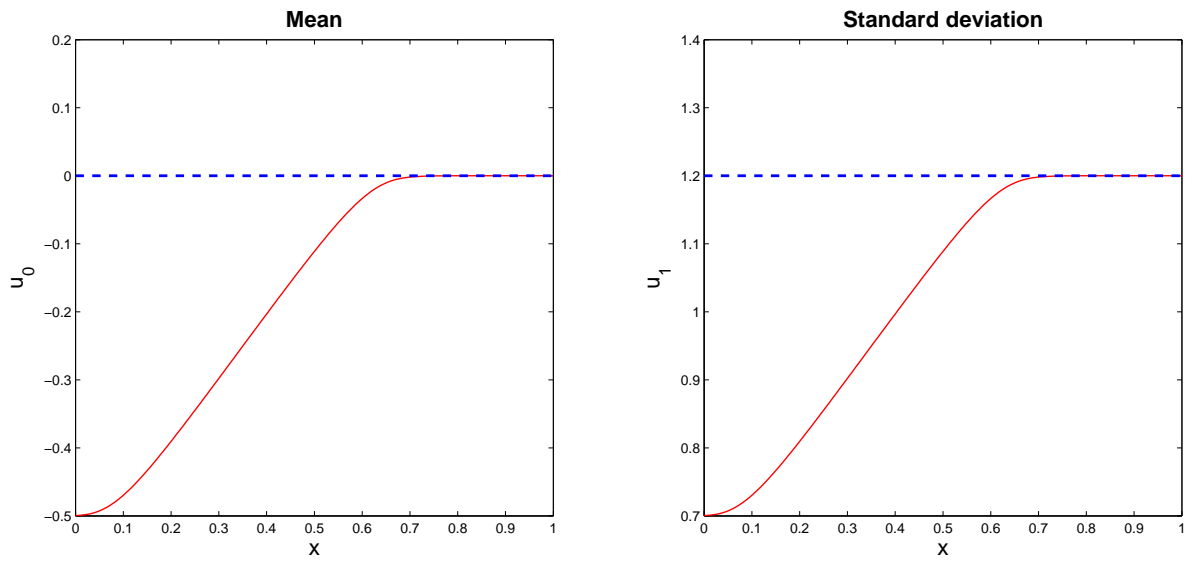

Figure 5: $u_{1}$ kept fixed at 0.2. $t=3$. Analytical solution: ---- ; Numerical solution: 

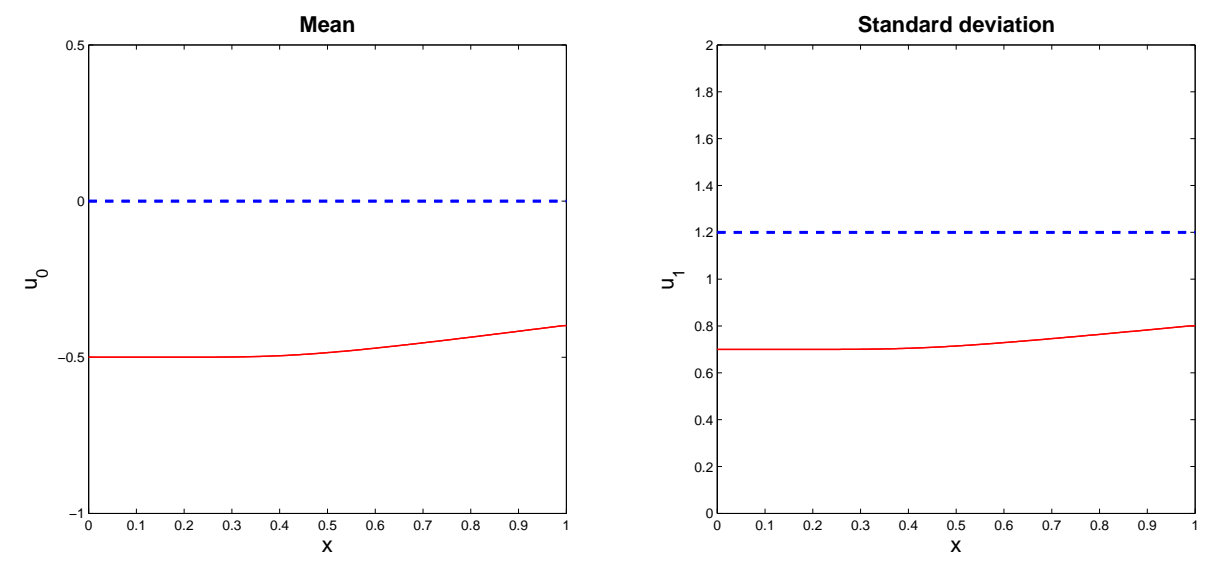

Figure 6: $u_{1}$ kept fixed at 0.2. $t=5$. Analytical solution: ---- ; Numerical solution:

\section{$5.4 u_{1}$ unknown at $x=0$, extrapolate $u_{1}$}

In this case the extrapolation $g_{1}=\left(u_{1}\right)_{1}$ is used to assign boundary data to the assumed unknown coefficient $u_{1}$. This case does not lead to stability using the energy method. As long as the analytical boundary conditions do not change, the numerical solution follows the analytical solution as before, see Figure 7. After $t=2.5$ the characteristics have reached the opposite boundaries and the error grows (Figure 8) before reaching the steady state (Figure 9).
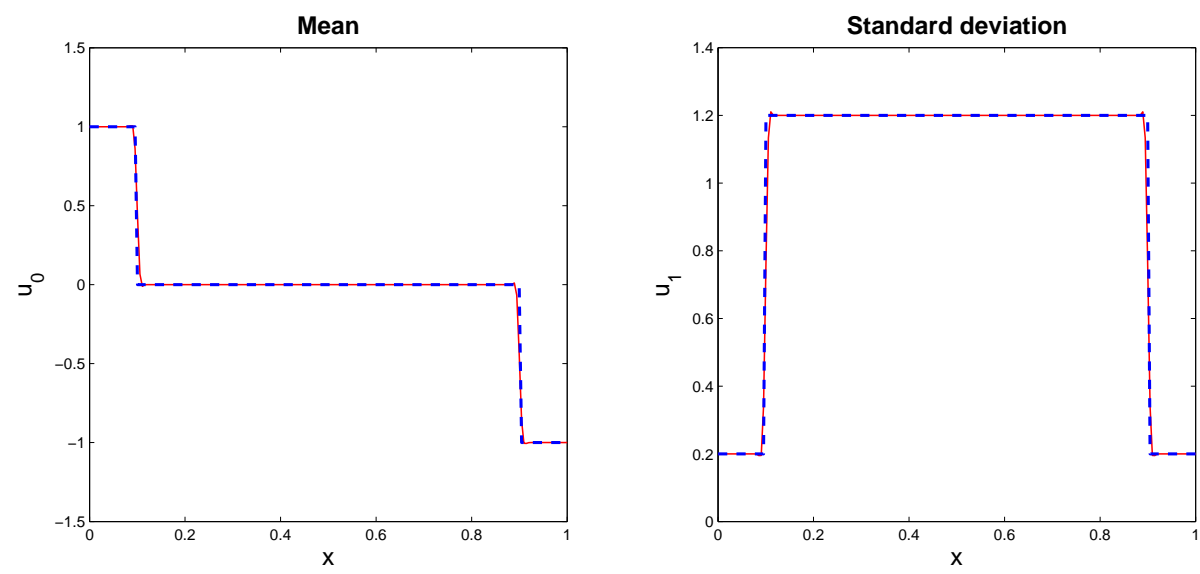

Figure 7: $u_{1}$ extrapolated from the interior. $t=2$. Analytical solution: ---- ; Numerical solution: - . 

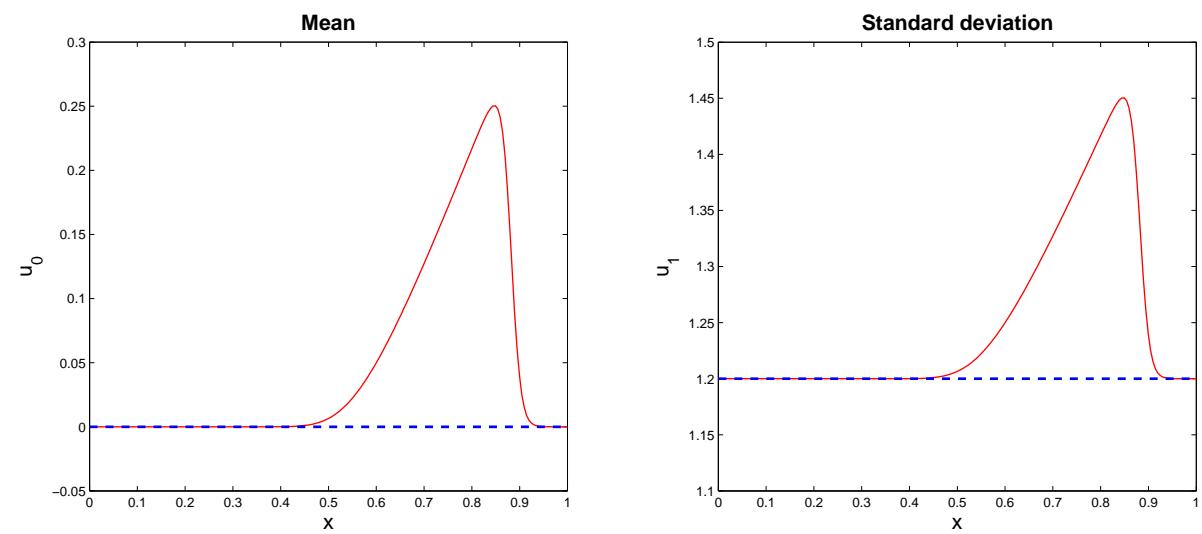

Figure 8: $u_{1}$ extrapolated from the interior. $t=3$. Analytical solution: ---- ; Numerical solution: $\longrightarrow$.
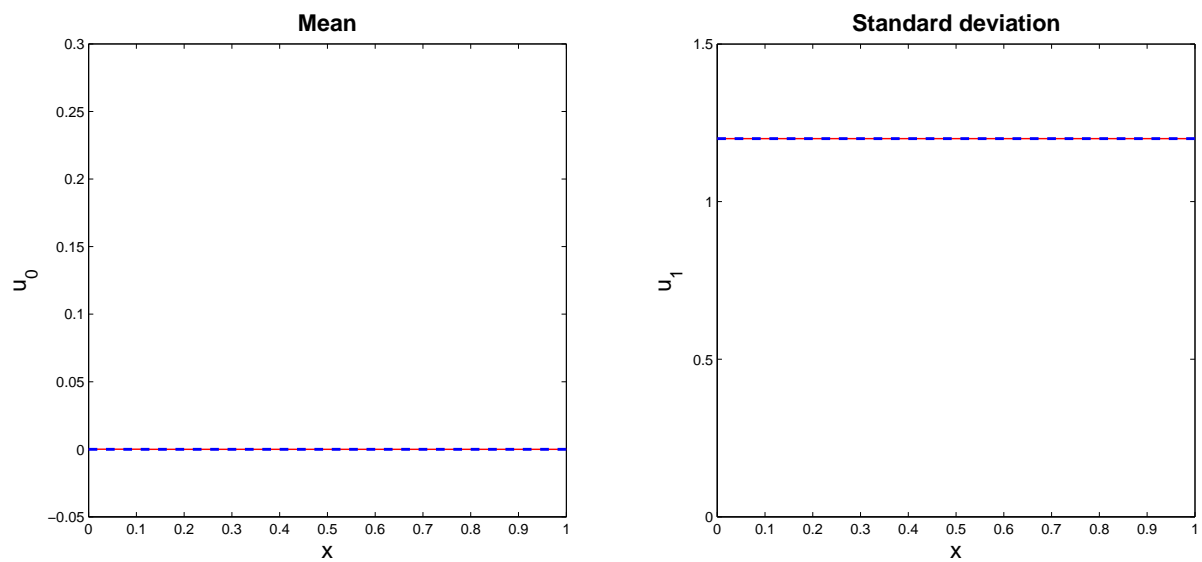

Figure 9: $u_{1}$ extrapolated from the interior. $t=5$. The error is of the order $10^{-15}$. Analytical solution: ---- ; Numerical solution: —— .

\section{$5.5 u_{0}$ unknown at $x=0$, guess $u_{0}$}

Next we assume that the boundary data for $u_{0}$ is unknown. This case leads to an energy estimate and stability. The same analysis is carried out for $u_{0}$ as was done for $u_{1}$ in the preceding section. First $u_{0}$ at $x=0$ is held fixed for all times. Figure 10 and Figure 11 show the solution before and after the true characteristics reach the boundaries. Note that the solution after a long time is not coincident with the analytical solution and that the boundary conditions are not satisfied (Figure 12). 

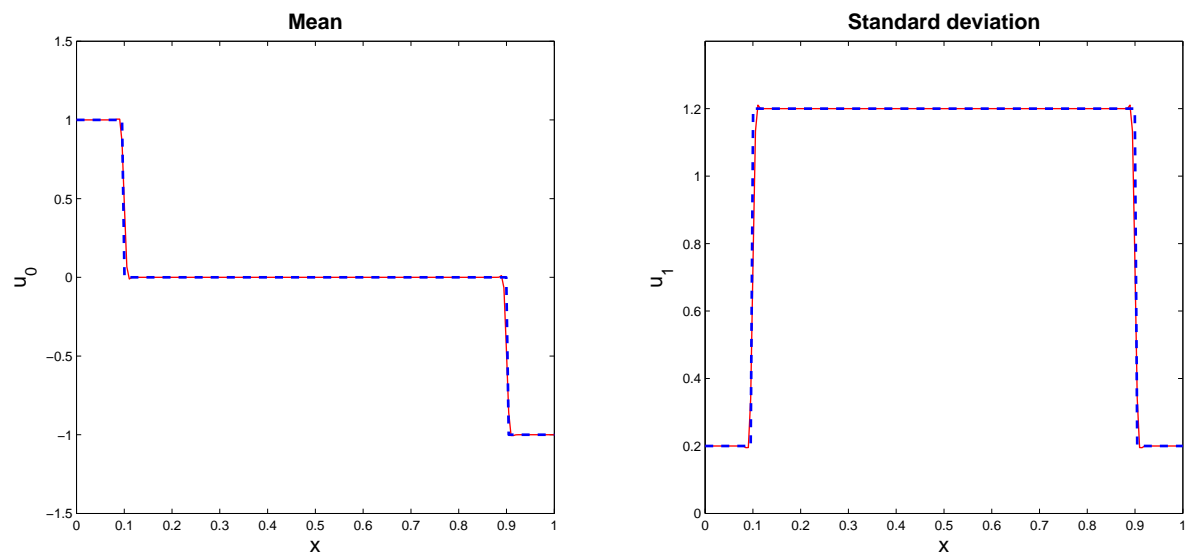

Figure 10: $u_{0}$ is held fixed. $t=2$. Analytical solution: ---- ; Numerical solution:
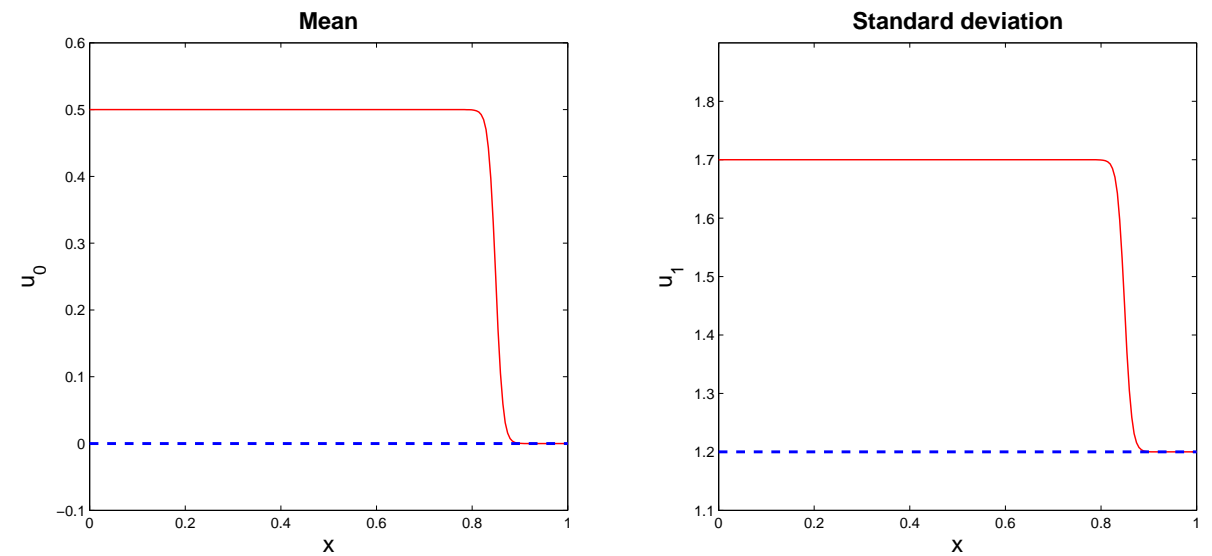

Figure 11: $u_{0}$ is held fixed. $t=3$. Analytical solution: ---- ; Numerical solution: 

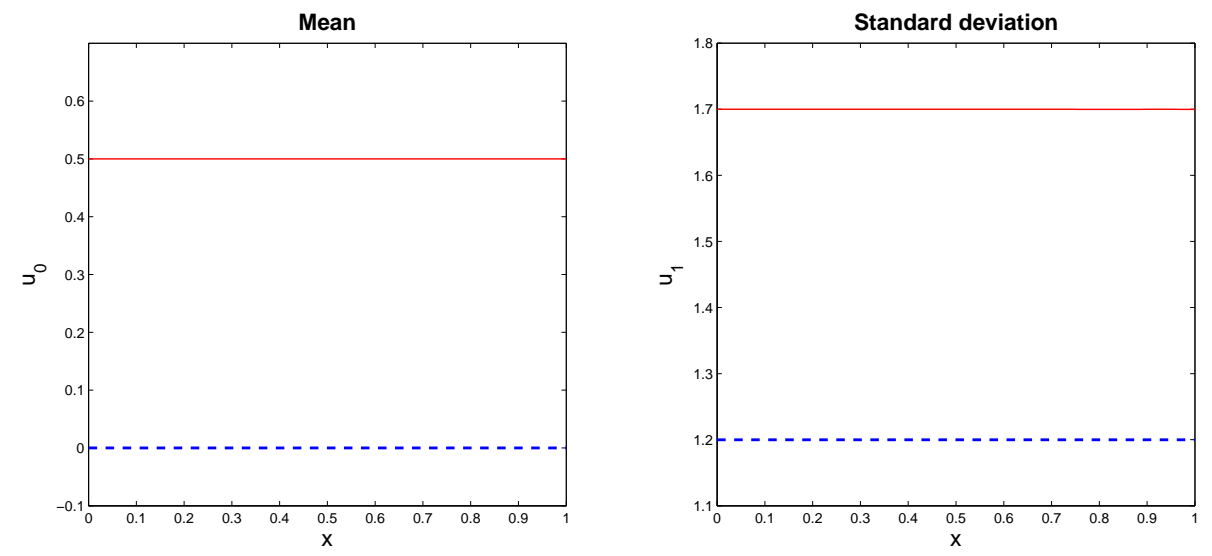

Figure 12: $u_{0}$ is held fixed. $t=5$. Analytical solution: ---- ; Numerical solution:

\section{6 $u_{0}$ unknown at $x=0$, extrapolate $u_{0}$}

The data for $u_{0}$ can be obtained from the interior of the domain. The extrapolation $g_{0}=\left(u_{0}\right)_{1}$ is used, see Figures 13, 14 and 15. This case does not lead to stability using the energy method. Note that the solution after a long time is very close to the analytical solution (Figure 15).
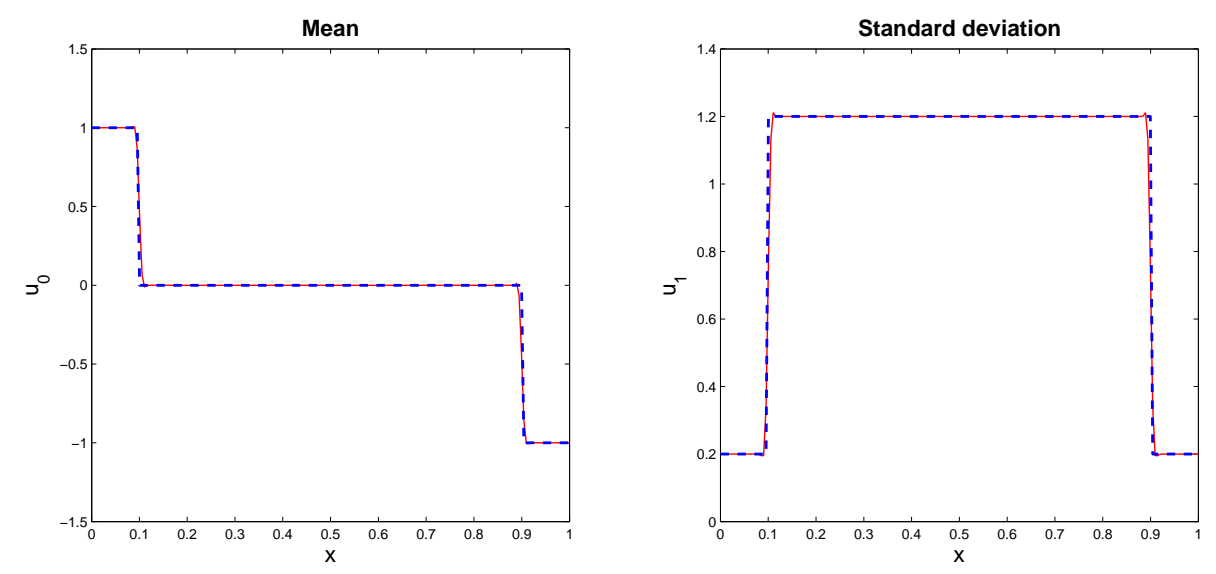

Figure 13: $u_{0}$ extrapolated from the interior. $t=2$. Analytical solution: ---- ; Numerical solution: — . 

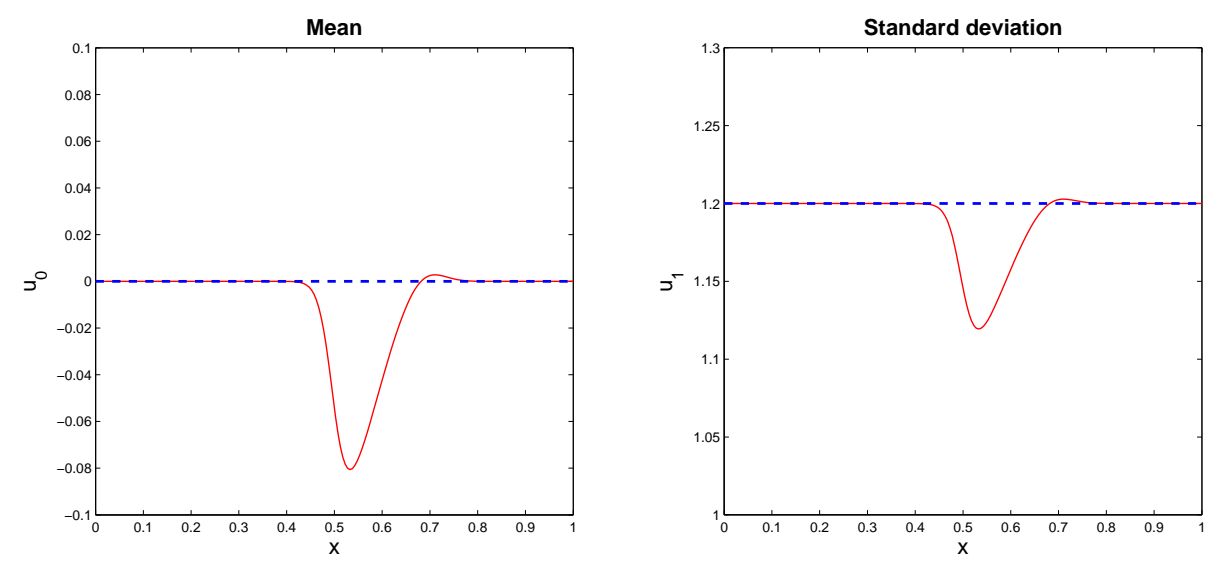

Figure 14: $u_{0}$ extrapolated from the interior. $t=3$. Analytical solution: ---- ; Numerical solution:
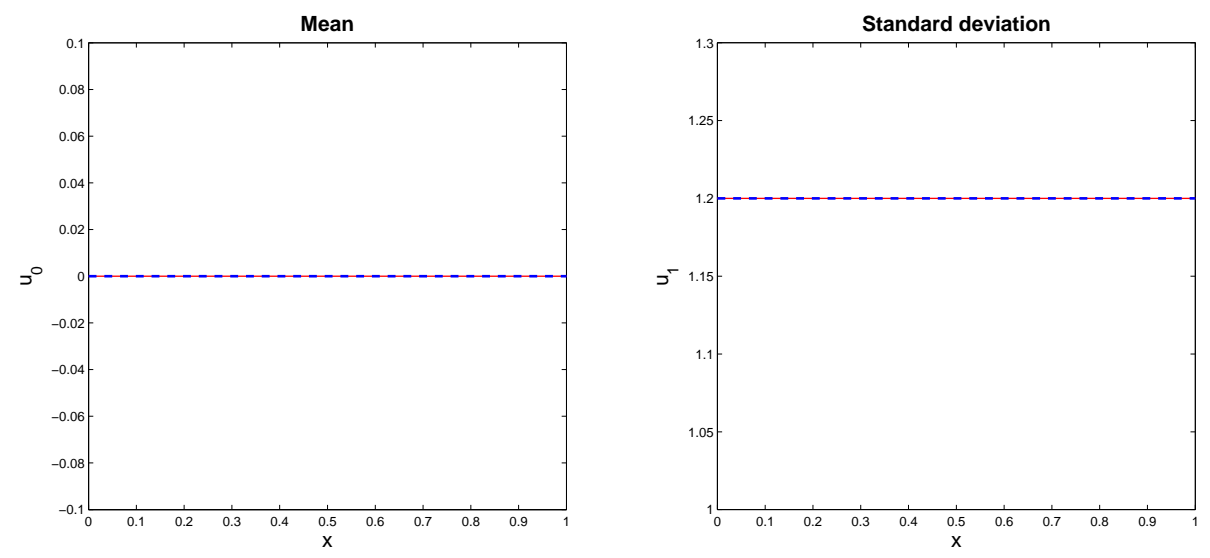

Figure 15: $u_{0}$ extrapolated from the interior. $t=5$. Analytical solution: ---- ; Numerical solution: —

\section{Conclusions}

The stochastic Galerkin method has been presented for Burgers' equation with stochastic boundary conditions. The analytical solution to the stochastic problem is smooth whereas the truncated system resulting from the Galerkin projection is discontinuous and subject to relatively slow convergence. We have shown how to obtain a well-posed and stable problem if we have perfect knowledge of the boundary data.

On the other hand, limited information at the boundaries lead surprising results. First of all, excellent results at steady state (for long time integration) are obtained 
using the extrapolation technique. This is probably due to the fact that only one boundary value is needed at the left boundary for $t>2.5$. Also, by guessing data of the mean value and the variance, equally poor results are obtained. The higher order modes might be very important. The order of the error obtained here indicate that appropriate approximation of the higher order terms is as important as guessing the expectation to get accurate results.

In many problems, sufficient data is not available to specify the correct number of variables. Unknown boundary values can then be constructed by extrapolation from the interior or by simply guessing the boundary data. We have investigated these two possible cases and for this specific problem the extrapolation technique based on penalty techniques was superior.

\section{References}

[1] Mark H. Carpenter, David Gottlieb, and Saul Abarbanel. Time-stable boundary conditions for finite-difference schemes solving hyperbolic systems: methodology and application to high-order compact schemes. J. Comput. Phys., 111(2):220-236, 1994.

[2] Mark H. Carpenter, Jan Nordström, and David Gottlieb. A stable and conservative interface treatment of arbitrary spatial accuracy. J. Comput. Phys., 148(2):341365, 1999.

[3] C. Chauvière, J. S. Hesthaven, and L. Lurati. Computational modeling of uncertainty in time-domain electromagnetics. SIAM J. Sci. Comput., 28(2):751-775, 2006 .

[4] Mike Christie, Vasily Demyanov, and Demet Erbas. Uncertainty quantification for porous media flows. J. Comput. Phys., 217(1):143-158, 2006.

[5] R. Ghanem, A. Doostan, and J. Red-Horse. A probabilistic construction of model validation. Computer Methods in Applied Mechanics and Engineering., 197:2585$2595,2008$.

[6] D. Gottlieb and J. S. Hesthaven. Spectral methods for hyperbolic problems. J. Comput. Appl. Math., 128(1-2):83-131, 2001.

[7] Beril Gustafsson, Heinz-Otto Kreiss, and Joseph Oliger. Time dependent problems and difference methods. Wiley, first edition, 1995.

[8] Ken Mattsson, Magnus Svärd, and Jan Nordström. Stable and accurate artificial dissipation. Journal of Scientific Computing, 21(1):57-79, 2004. 
[9] Jan Nordström. Conservative finite difference formulations, variable coefficients, energy estimates and artificial dissipation. J. Sci. Comput., 29(3):375-404, 2006.

[10] Jan Nordström and Mark H. Carpenter. Boundary and interface conditions for high-order finite-difference methods applied to the Euler and Navier-Stokes equations. J. Comput. Phys., 148(2):621-645, 1999.

[11] Jan Nordström and Mark H. Carpenter. High-order finite difference methods, multidimensional linear problems, and curvilinear coordinates. J. Comput. Phys., 173(1):149-174, 2001.

[12] Per Pettersson, Gianluca Iaccarino, and Jan Nordström. Numerical analysis of the Burgers equation in the presence of uncertainty. Journal of Computational Physics, 228:8394-8412, 2009.

[13] S. Poroseva, J. Letschert, and M. Y. Hussaini. Uncertainty quantification in hurricane path forecasts using evidence theory. APS Meeting Abstracts, pages B1+, 2005.

[14] Matthew T. Reagan, Habib N. Najm, Roger G. Ghanem, and Omar M. Knio. Uncertainty quantification in reacting-flow simulations through non-intrusive spectral projection. Combustion and Flame, pages 545-555, 2003.

[15] Y. Yu, M. Zhao, T. Lee, N. Pestieau, W. Bo, J. Glimm, and J. W. Grove. Uncertainty quantification for chaotic computational fluid dynamics. J. Comput. Phys., 217(1):200-216, 2006. 